\title{
Upregulation of miR-144-3p expression attenuates glioma cell viability and invasion by targeting BCL6
}

\author{
JINGRU ZHOU ${ }^{1}$ and RUEN LIU ${ }^{2}$ \\ ${ }^{1}$ Department of Neurosurgery, Nanchang University People's Hospital, Nanchang, Jianxi 330006; \\ ${ }^{2}$ Department of Neurosurgery, Peking University People's Hospital, Beijing 100044, P.R. China
}

Received June 2, 2020; Accepted October 14, 2020

DOI: 10.3892/etm.2021.10591

\begin{abstract}
Glioma remains to be an aggressive type of cancer with poor prognosis irrespective of the type of standard treatment applied. Therefore, identification of accurate early diagnostic methods and therapeutic strategies for glioma is imperative for the treatment of this disease. The expression of a number of miRNAs in glioma have been reported to be associated with the regulation of tumorigenic progression, cancer cell proliferation, metastasis, invasion, angiogenesis and drug resistance. The aim of the present study was to assess the function of the microRNA (miR/miRNA)-144-3p/BCL6 axis in glioma. Reverse transcription-quantitative PCR was used to measure miR-144-3p and BCL6 expression. Western blotting was used for measuring BCL6 expression. Luciferase reporter assay was used to assess the association between miR-144-3p and BCL6 and a tumor xenograft model was established for assess tumor growth. The data demonstrated that miR-144-3p was decreased whereas BCL6 expression was increased in glioma tissues compared with those in healthy human brain tissues, where miR-144-3p suppressed BCL6 expression by targeting the 3'-UTR sequence of BCL6. miR-144-3p overexpression alleviated proliferation and invasion in $\mathrm{U} 251$ cells whereas transfection with the BCL6-overexpressing plasmid rescued the suppressive effects of miR-144-3p upregulation on the proliferation and invasion of U251 cells. In addition, miR-144-3p overexpression and BCL6 downregulation inhibited tumor progression in a mouse tumor xenograft model. The present findings suggest that miR-144-3p and BCL6 may serve to be indicator of proliferation and invasion for patients with glioma. Furthermore, BCL6 may serve an important role in
\end{abstract}

Correspondence to: Professor Ruen Liu, Department of Neurosurgery, Peking University People's Hospital, 11 South Xizhimen Street, Beijing 100044, P.R. China

E-mail:1repku@sina.com

Dr Jingru Zhou, Department of Neurosurgery, Nanchang University People's Hospital, 152 Aiguo Road, Nanchang, Jianxi 330006, P.R. China

E-mail:jrzhoupk@sina.com

Key words: glioma, microRNA-144-3p, BCL6, viability, invasion the miR-144-3p-mediated regulation of proliferation and invasion of glioma cells, where the miR-144-3p/BCL6 axis can be used to target patients with glioma therapeutically.

\section{Introduction}

Glioma remains to be an aggressive type of brain tumor with limited improvement to its diagnosis and treatment methoods over the past decades $(1,2)$. The molecular biology underlying glioma is complex, which obstructs the development of specific diagnostic and treatment strategies $(3,4)$. The dysregulation of a number of signaling pathways, including the Epidermal growth factor receptor/AKT/PTEN, receptor tyrosine kinase $(\mathrm{RTK}) / \mathrm{PI} 3 \mathrm{~K}, \mathrm{Wnt} / \beta$-catenin and $\mathrm{NF}-\kappa \mathrm{B}$ pathways has been shown to occur during the development of glioma (4-6). Dysregulated RTK, RB and tumor protein p53 (TP53) pathways in glioma have been highlighted by previous genomic analyses $(7,8)$. However, the mechanism promoting the aggressive pathophysiology of glioma remain unclear.

BCL6 is an important regulator of the germinal center response and protooncogene expression in diffuse large B-cell lymphoma (9). This protein has been reported to serve an important role in the pathology of multiple tumors and diseases, including breast cancer, ovarian cancer and glioma (10-12). It has been previously shown that BCL6 exprssion is regulated by transcription factors such as STAT3 and STAT5B and epigenetic modifications, including DNA methylation and histone modifications (13). Furthermore, BCL6 has been proposed to be a prognostic marker and a targetable glioma-promoting factor (12) whereby knocking down he expression of this protein can inhibit the pathophysiology of glioma cells and trigger cell apoptosis (12). However, the precise role of the BCL6 regulatory network within glioma requires additional studies.

microRNA (miR or miRNA)-mediated regulation of BCL6 expression has been previously documented to serve a role in tumor progression of breast cancer, by targeting the 3'-untranslated region (3'UTR) sequence of BCL6 mRNA (14). miRNA-187-3p, miR-519d-3p and miR-554 can also suppress cell proliferation and invasion by downregulating BCL6 expression in gastric, non-small cell lung and breast cancers (14-16). miRNAs are a class of small non-coding RNAs that can bind to target mRNAs to regulate gene expression on a post-transcriptional level $(17,18)$. Previous expression 
studies on specific miRNAs have reported their association with the pathology and prognosis of glioma (19-21). The different expression profile of miRNAs in glioma compared with healthy brain tissues implicate their role in the regulation of tumorigenic progression, possibly in processes including glioma proliferation, metastasis, invasion, angiogenesis and drug resistance (20). The development of glioma is associated with a significant reduction in the levels of antitumor miRNAs, such as miR-7 and miR-137 (22). By contrast, the opposite trend is noted with regards to the expression levels of the protooncogenic miRNAs, including miR-10b, miR-21, miR-182, miR-222 and miR-423-5p, all of which have been previously demonstrated to promote tumorigenesis and development of glioma $(20,21)$.

In the present study, the ability of miR-144-3p to act as a suppressor of glioma development was investigated by using reverse transcription-quantitative PCR (RT-qPCR), western blotting and luciferase reporter assays and a tumor xenograft model. These findings may provide a new and potential treatment strategy for glioma.

\section{Materials and methods}

Tissue collection. A total of 25 glioma tissues were obtained from who underwent surgical resection and 10 normal human brain tissues (mean age, $54.74 \pm 6.52$ years; 3 female and 7 male) were obtained from patients with brain injury who underwent partial resections of brain tissues to reduce intracranial pressure were collected from individuals at the Peking University People's Hospital (Beijing, China) between February 2017 and January 2018. The mean age of patients was $60.36 \pm 8.81$ years ( $\geq 60$ years, $n=16 ;<60, n=9$ ), including 11 female and 14 male. The inclusion criteria were that all glioma samples had confirmed pathological diagnosis and patients who did not receive any other therapies before surgery. Patients who with complications, such as other malignant tumors, serious systemic infections and other severe systemic diseases, were excluded. The tissues were rapidly placed in liquid nitrogen and subsequently stored at $-80^{\circ} \mathrm{C}$. An aliquot of the tissue was pathologically examined prior to further examination. All participants met the following inclusion criteria that no preoperative radiotherapy or chemotherapy was performed. The clinical samples were collected after written informed consent was obtained from each participant and after the study was authorized by the Ethics Committee of Peking University People's Hospital (Beijing, China).

Cell culture. The glioma U251 cell line was purchased from the Cell Bank of Type Culture Collection of the Chinese Academy of Sciences. U251 cells were cultured in DMEM (Invitrogen; Thermo Fisher Scientific, Inc.) with 10\% FBS (Invitrogen; Thermo Fisher Scientific, Inc.) and $1 \%$ penicillin-streptomycin (Gibco; Thermo Fisher Scientific, Inc.) at $37^{\circ} \mathrm{C}$ in a humidified atmosphere in the presence of $5 \% \mathrm{CO}_{2}$.

Transfection assay. BCL6-overexpressing (pcDNA3.1, Cyagen Biosciences, Inc.; NCBI Accession Gene ID: 604; https:// www.ncbi.nlm.nih.gov/nuccore/NM_001130845.1). Total RNA was extracted from U251 cells and reversed transcribed into cDNA as a template for PCR amplification (forward,
5'-CCGGAATTCATGGCCTCGCCGGCTGACAGCTGT-3' and reverse, 5'-CCCAAGCTTTCAGCAGGCTTTGGGGAG CTC-3'; Ultra HiFidelity PCR Kit, cat. no. KP203, Tiangen Biotech Co., Ltd.; thermocycling conditions, $94^{\circ} \mathrm{C}$ for $3 \mathrm{~min}$, followed by 30 cycles of $94^{\circ} \mathrm{C}$ for $30 \mathrm{sec}, 60^{\circ} \mathrm{C}$ for $30 \mathrm{sec}$ and $72^{\circ} \mathrm{C}$ for $5 \mathrm{~min}$ ). After the BCL6 target gene was obtained, it was ligated and recombined with pcDNA3.1 vector. BCL6 short hairpin (sh) RNA (5'-tcgagtgctgttgacagtgagcgaGCCTGTTCT ATAGCATCTTTAtagtgaagccacagatgtaTAAAGATGCTAT AGAACAGGCgtgcetactgcctcggaa-3') plasmid (pcDNA3.1), the corresponding negative control (NC) plasmid (NC-shRNA, 5'-TTCTCCGAACGTGTCACGT-3'), miR-144-3p mimic (5'-UCAUGUAGUAGAUAUGACAU-3'), miR-144-3p NC (5'-UUCUCGAACGUGUCACGUUUU-3'), miR-144-3p inhibitor (5'-AUGUCAUAUCUACUACAUGA-3'), and inhibitor NC (5'-CAGUACUUUUGUGUAGUACAA-3') were obtained from Cyagen Bioscience, Inc. A mixed solution containing the plasmid $(2 \mu \mathrm{g})$ and miRs $(20 \mathrm{nM})$ and Lipofectamine $^{\circledR} 3000$ (Invitrogen; Thermo Fisher Scientific, Inc.) was diluted with serum-free DMEM. According to the manufacturer's protocols, the transfection mixture was added to the cell suspension and replaced with complete medium following incubation for $8 \mathrm{~h}$ at $37^{\circ} \mathrm{C}$. Subsequent experiments were conducted following $48 \mathrm{~h}$ of cell culture.

Luciferase reporter assay. Bioinformatic prediction were conducted by starBase v2.0 (http://starbase.sysu.edu. cn/agoClipRNA.php?source $=m R N A)$ and TargetScanHuman v7.2 (http://www.targetscan.org/vert_72/). The pMIR-BCL6 Luciferase vector (Promega Corporation) was prepared using the 3'-UTR sequence of BCL6. To generate the reporter vectors containing the potential binding sites of miR-144-3p, the DNA fragments containing the 3'UTR of target the gene BCL6 (NCBI Accession Gene ID: 604; https://www.ncbi.nlm.nih. gov/nuccore/NM_001130845.1) were amplified (3'-UTR BCL6 forward, 5'-GTTGATGCTTTCGTCTCCAGC-3' and reverse, 5'-ATCCCATGATGTAGTGCCTCT-3'). Ultra Super High Fidelity PCR Kit (cat. no. KP203, Tiangen Biotech Co., Ltd.) was conducted from the cDNA extracted from U251 cells by following conditions: $94^{\circ} \mathrm{C}$ for $3 \mathrm{~min}$, followed by 30 cycles of $94^{\circ} \mathrm{C}$ for $30 \mathrm{sec}, 60^{\circ} \mathrm{C}$ for $30 \mathrm{sec}$ and $72^{\circ} \mathrm{C}$ for $5 \mathrm{~min}$. The fragments were then cloned into the pmirGLO luciferase reporter plasmid (Promega Corporation). Quick-Change Site-Directed Mutagenesis kit (cat. no. 210518; Stratagene; Agilent Technologies, Inc.) was used for gene mutation for the production of the pMIR-BCL6-Mut plasmid. Following the seeding of $5 \times 10^{3} \mathrm{U} 251$ cells into 24 -well plates, the cells were co-transfected with $200 \mathrm{ng}$ pMIR-BCL6 or pMIR-BCL6-Mut and $50 \mathrm{nM}$ miR-144-3p mimic or miR-control using the Lipofectamine ${ }^{\circledR}$ 3000 reagent (Invitrogen; Thermo Fisher Scientific, Inc.). Subsequently, the cells were harvested and lysed following $36 \mathrm{~h}$ of cell culture after transfection. The Dual-Luciferase ${ }^{\circledR}$ Reporter Assay System (Promega Corporation) was used for measuring Renilla luciferase and firefly luciferase activity using a Glomax ${ }^{\circledR}$ Luminometer (Promega Corporation). Normalized Renilla-luciferase values were represented relative to the control according to the manufacturer's protocol.

Reverse transcription-quantitative PCR (RT-qPCR). Total RNA was extracted from tissues and U251 cells using TRIzol ${ }^{\circledR}$ 
Reagent (Invitrogen; Thermo Fisher Scientific, Inc.) according to manufacturer's protocol. PrimeScript ${ }^{\mathrm{TM}} \mathrm{RT}$ reagent Kit (cat. no. RR037A; Takara Bio, Inc.) was used to reverse transcribe the cDNA starting from total RNA using the protocol of $37^{\circ} \mathrm{C}$ for $15 \mathrm{~min}, 85^{\circ} \mathrm{C}$ for $5 \mathrm{sec}$, and $4^{\circ} \mathrm{C}$ preservation. For mRNA and miR detection, $\mathrm{qPCR}$ experiments were conducted using the SuperReal PreMix Plus (SYBR Green; cat. no. FP205; Tiangen Biotech Co., Ltd.). qPCR was carried out under the following conditions: $95^{\circ} \mathrm{C}$ for $30 \mathrm{sec}$, followed by 35 cycles of $95^{\circ} \mathrm{C}$ for $5 \mathrm{sec}, 60^{\circ} \mathrm{C}$ for $30 \mathrm{sec}$ and $68^{\circ} \mathrm{C}$ for $5 \mathrm{~min}$. Each sample was analyzed in triplicate, and quantified using the $2^{-\Delta \Delta C q}$ method (23). The sequences of primers were as follows: BCL6 forward, 5'-GTGATGGCCACGGCTATGTA-3' and reverse, 5'-CATCCGGCTGTTGAGGAACT-3' and GAPDH (internal reference) forward, 5'-ACCATCTTCCAGGAGCGAGA-3' and reverse, 5'-GACTCCACGACGTACTCAGC-3'.

miRNA qPCR. The miRcute Plus miRNA qPCR Kit (cat. no. FP411; Tiangen Biotech Co., Ltd.) was used to measure miR-144-3p levels. Following reverse transcription ( $42^{\circ} \mathrm{C}$ for $60 \mathrm{~min}, 95^{\circ} \mathrm{C}$ for $3 \mathrm{~min}$ and $4^{\circ} \mathrm{C}$ preservation) by the miRcute Plus miRNA First-Strand cDNA Kit (cat. no. KR211; Tiangen Biotech Co., Ltd.), RT-qPCR was conducted by a miR-144-3p forward primer and a universal reverse primer. PCR reaction conditions were: Pre-denaturation at $95^{\circ} \mathrm{C}$ for $15 \mathrm{~min}$, followed by 40 cycles of denaturation at $94^{\circ} \mathrm{C}$ for $20 \mathrm{sec}$ and annealing and extension at $60^{\circ} \mathrm{C}$ for $34 \mathrm{sec}$. Each sample was analyzed in triplicate, and quantified using the $2^{-\Delta \Delta \mathrm{Cq}}$ method (23). The primers were as follows: hsa-miR-144-3p, 5'-CCCTACAGTATAGATGATGTA-3'; and U6 small nuclear RNA (internal reference), 5'-CAAATT CGTGAAGCGTTCCATAT-3'. The universal reverse primers of hsa-miR-144-3p and U6 were purchased from Tiangen Biotech Co., Ltd (cat. no. FP411).

Western blotting analysis. The U251 cells were first lysed using RIPA lysis buffer and protein concentrations were quantified by using a BCA kit (Thermo Fisher Scientific, Inc.), before being subsequently boiled at $100^{\circ} \mathrm{C}$ for $5 \mathrm{~min}$ and stored at $-20^{\circ} \mathrm{C}$. In total, $30 \mu \mathrm{g}$ proteins were separated by $10 \%$ SDS-PAGE gels and transferred onto PVDF membranes, which were blocked with $5 \%$ milk in TBST $(0.05 \%$ Tween 20$)$ for $1 \mathrm{~h}$ at room temperature. The membranes were incubated with the following primary antibodies at $4^{\circ} \mathrm{C}$ overnight: Anti-BCL6 (cat. no. ab33901; 1:1,000; Abcam) and anti-GAPDH (cat. no. ab181602; 1:2,000; Abcam). The following morning, the membranes were incubated with HRP-linked secondary antibody (cat. no. ASR1089; 1:20,000; Abcepta Biotech Co., Ltd.) for $1 \mathrm{~h}$ at room temperature. The bands were visualized using an enhanced chemiluminescence detection system (cat. no. 1705060; Bio-Rad Laboratories, Inc.). Optical densities of the bands were calculated using a MiVnt image analysis system (Version 5.2.1; Bio-Rad Laboratories, Inc.).

Cell Counting Kit-8(CCK-8) assay. Cell viability was assessed using the CCK8 assay. U251 cells were seeded at a density of $3 \times 10^{3}$ cells in 96-well plates before being transfected with the miR-144-3p mimic (or NC) or inhibitor (or inhibitor NC) and cultured for $12,24,48$ or $72 \mathrm{~h}$ at $37^{\circ} \mathrm{C}$. A total of $10 \mu \mathrm{l} \mathrm{CCK}-8$ solution (Dojindo Molecular Technologies, Inc.) was added into the culture medium in each well and incubated for $1 \mathrm{~h}$ at $37^{\circ} \mathrm{C}$. The microplate reader (Thermo Fisher Scientific Inc.) was then used for measuring the optical density in each well at $450 \mathrm{~nm}$.

Cell invasion assay. Transwell assay was conducted using a Transwell chamber ( $8 \mu \mathrm{m}$; cat. no. 3422; Corning, Inc.) pre-coated with Matrigel Matrix (Matrigel 1:8 dilution with DMEM put onto the upper compartment surface of the membrane at the bottom of the Transwell and incubated at $37^{\circ} \mathrm{C}$ for $2 \mathrm{~h}$; BD Biosciences) at $37^{\circ} \mathrm{C}$. The bottom chamber of the wells contained $750 \mu \mathrm{l}$ DMEM with $10 \%$ FBS whereas the top chamber contained $250 \mu \mathrm{l}$ cell suspension $\left(1 \times 10^{5} / \mathrm{ml}\right.$ with $2 \%$ FBS). Following culture of the cells at $24 \mathrm{~h}$ at $37^{\circ} \mathrm{C}$, the cells that failed to invade were removed from the top chamber and subsequently the cells on the membrane were stained with $0.5 \%$ crystal violet for $15 \mathrm{~min}$ at room temperature. The images were obtained from three independents fields (magnification, $\mathrm{x} 10$ )/well using an inverted light microscope (Olympus Corporation). Following culture of the cells at $24 \mathrm{~h}$ at $37^{\circ} \mathrm{C}$, the cells that failed to invade were removed from the top chamber and subsequently the number of cells on membrane were measured by the CCK- 8 assay. The added CCK- 8 solution was used to determine the relative cell invasive capacity by measuring the OD at $450 \mathrm{~nm}$.

Tumor xenograft model. The in vivo animal assay was authorized by the Institutional Animal Care and Use Committee and the Local Ethics Board of the Peking University People's Hospital (Beijing, China). In total, 6-8 week old male nude mice $(n=24)$ were purchased from Beijing Vital River Laboratory Animal Technology Co., Ltd and fed under specific pathogen free conditions, in the mouse-feeding facility with a 12 -h light/dark cycle at $22 \pm 2^{\circ} \mathrm{C}$ and $50-60 \%$ humidity, with free foraging and activity and free access to food and water. Following transfection for $48 \mathrm{~h}$ with a final transfection concentration of $20 \mathrm{nM}$ miR-144-3p mimic, miR-NC, BCL6-shRNA or NC-shRNA ( $n=6 /$ group), U251 cells $\left(2 \times 10^{6}\right.$ in serum-free DMEM, $200 \mu \mathrm{l}$ ) were subcutaneously injected into the flanks of the mice. Following implantation, tumor nodules appeared at the initial site of injection after $\sim 7$ days. The tumor volume was measured, including length and width. The tumor volume (TV) was calculated very week, according to the following formula: TV $\left(\mathrm{mm}^{3}\right)=1 / 2 \times$ width $^{2} \mathrm{x}$ length. The tumor-burdened nude mice were sacrificed 28 days following tumor implantation and the tumor tissues were obtained. The health of the mice was monitored daily and all mice were sacrificed by dislocation of the cervical vertebrae when symptoms of terminal tumor burden were observed, including tumor volume $>1,000 \mathrm{~mm}^{3}$, $>20 \%$ weight loss, hunched posture, cranial protrusion and significant neurologic symptoms (seizures and abnormal gait or posture), which developed dyspnea. No animal reached the criteria before these endpoints.

Statistical analysis. All data are presented as mean \pm SEM from four indepdent experiments. The SPSS version 22.0 (IBM Corp.) was used for all statistical analyses, which was conducted using two-tailed Student's t-test or one-way ANOVA with least significant difference post hoc test. $\mathrm{P}<0.05$ was considered to indicate a statistically significant difference. 
A

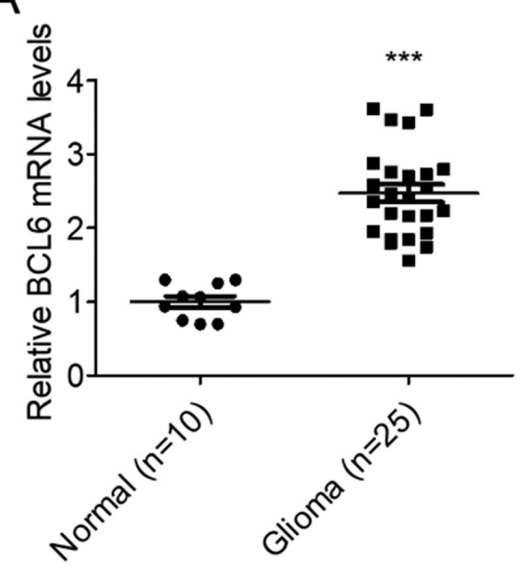

$\mathrm{B}$

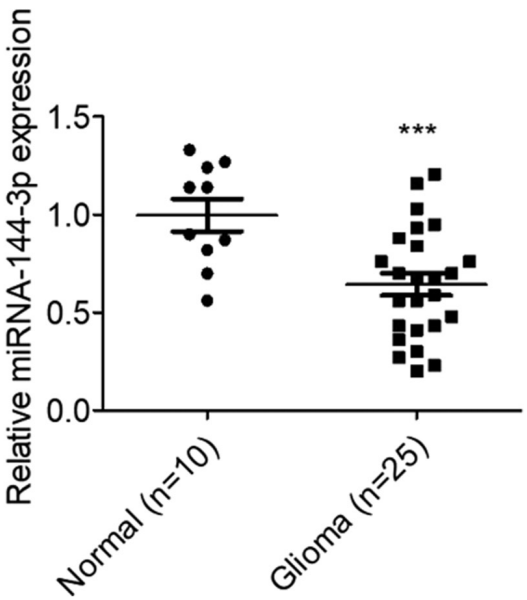

Figure 1. miR-144-3p and BCL6 expression in glioma tisseus. Reverse transcription-quantitative PCR was used for the measurement of the relative (A) miR-144-3p and (B) BCL6 expression in 25 glioma and 10 normal brain tissues. ${ }^{* * *} \mathrm{P}<0.001$ vs. Normal. miR, microRNA.

\section{Results}

Downregulation of miR-144-3p expression and upregulation of BCL6 expression in glioma tissues. To assess miR-144-3p expression on glioma, its levels were measured in 25 glioma tissues and 10 normal brain tissues. RT-qPCR data suggested that miR-144-3p expression was significantly reduced, whilst BCL6 expression was significantly increased in glioma tissues compared with those in the normal brain tissues $(\mathrm{P}<0.001$; Fig. 1). These results suggest that miR-144-3p and BCL6 are involved in glioma development.

miR-144-3p suppresses BCL6 expression by targeting its 3'-UTR sequence in U251 cells. Bioinformatic prediction and targetscan demonstrated that BCL6 could be a potential target gene of miR-144-3p (Fig. 2A). To verify the association between miR-144-3p and BCL6, the pMIR vector was packaged with WT or MUT BCL6 3'-UTR sequences before luciferase reporter assay was performed in U251 cells. The data indicated that luciferase activity was significantly downregulated in glioma U251 cells co-transfected with miR-144-3p mimic and WT BCL6 3'-UTR compared with that in the WT control group $(\mathrm{P}<0.001)$. However, luciferase activity levels were unaltered in U251 cells transfected with MUT BCL63'-UTR (Fig. 2B). Therefore, the data confirmed that BCL6 is a target gene for miR-144-3p in glioma cells.

The role of miR-144-3p on BCL6 expression was next investigated following transfection with the miR-144-3p mimic or inhibitor into U251 cells. Transfection with the miR-144-3p mimic significantly upregulated miR-144-3p levels whereas transfection with the miR-144-3p inhibitor significantly decreased miR-144-3p expression ( $\mathrm{P}<0.001$; Fig. 3A). Western blotting analysis of BCL6 protein levels was next performed. The protein expression levels of BCL6 were significantly downregulated following transfection of the cells with the miR-144-3p mimic, whilst they were enhanced following miR-144-3p knockdown in U251 cells compared with those in the control group $(\mathrm{P}<0.001$; Fig. 3B).

miR-144-3p reduces U251 cell proliferation and invasion in vitro. CCK-8 assay demonstrated that upregulation of miR-144-3p significantly reduced U251 cell viability whereas miR-144-3p

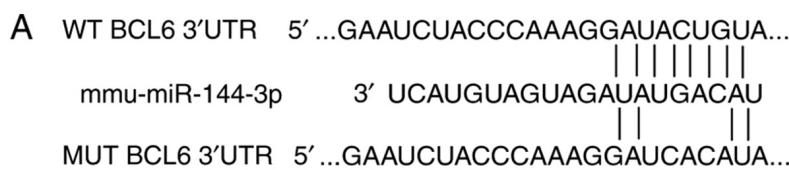

MUT BCL6 3'UTR 5 5'...GAAUCUACCCAAAGGAUCACAUA...

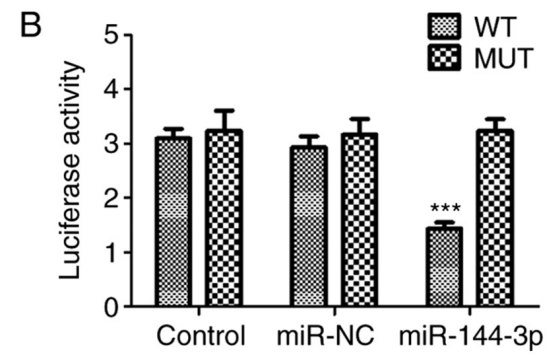

Figure 2. BCL6 is a target of miR-144-3p. (A) Targetscan software was used to predict that BCL6 is a target of miR-144-3p where the binding sequences of WT or MUT BCL6 3'-UTR for miR-144-3p are shown. (B) Assessment of luciferase activity following transfection with WT or MUT BCL6 3'UTR and miR-NC or miR-14-3p mimics using a Glomax luminometer and subsequent normalization. All data are presented as the mean $\pm \operatorname{SEM}(n=4) .{ }^{* * *} \mathrm{P}<0.001$ vs. the control-WT group. WT, wild-type; MUT, mutant; 3'-UTR, 3' untranslated region; miR, microRNA.

knockdown significantly increased U251 cell viability compared with that in control $(\mathrm{P}<0.001$; Fig. $4 \mathrm{~A})$. The Transwell assay results suggested that increasing miR-144-3p expression levels significantly suppressed U251 cell invasion compared with that in control, whilst miR-144-3p inhibition increased U251 cell invasion compared with that in the control ( $\mathrm{P}<0.001$; Fig. 4B).

BCL6 lie downstream of miR-144-3p-mediated glioma cell proliferation and invasion in vitro. To examine the downstream effects of miR-144-3p on glioma cell viability and invasion, miR-144-3p-overexpressing U251 cells were co-transfected with a BCL6-activation plasmid. western blotting analysis was first performed to measure the effects of co-transfection on BCL6 expression. The transfection efficiency of pcDNA3.1-BCL6 and BCL6-shRNA was assessed by RT-qPCR, where pcDNA3.1-BCL6 upregulated BCL6 expression and BCL6-shRNA inhibited BCL6 expression compared wth that in the control group (Fig. S1). BCL6 expression levels 

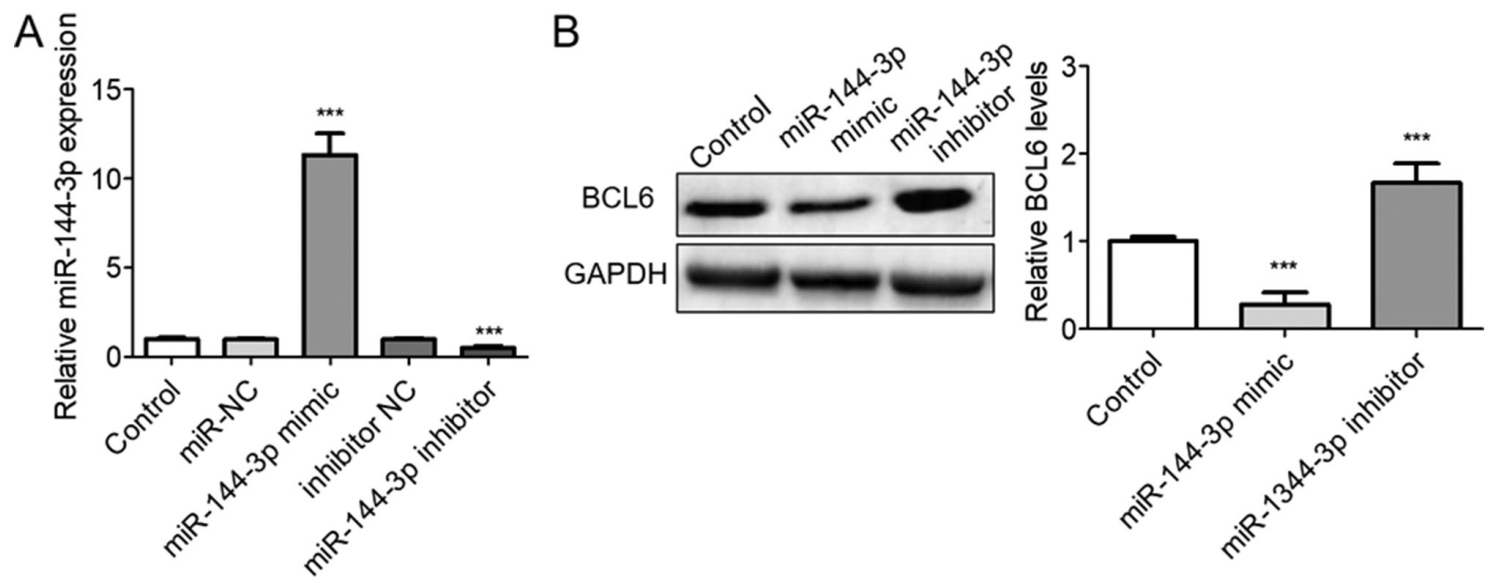

Figure 3. miR-144-3p overexpression suppresses BCL6 expression. (A) Reverse transcription-quantitative PCR analysis was used to measure miR-144-3p expression in miR-144-3p mimic- or inhibitor-transfected U251 cells. (B) Western blot analysis was conducted to examine BCL6 protein expression following transfection. All data are presented as the mean $\pm \operatorname{SEM}(n=4) .{ }^{* * * *} \mathrm{P}<0.001$ vs. Control (untransfected). miR, microRNA; NC, negative control.
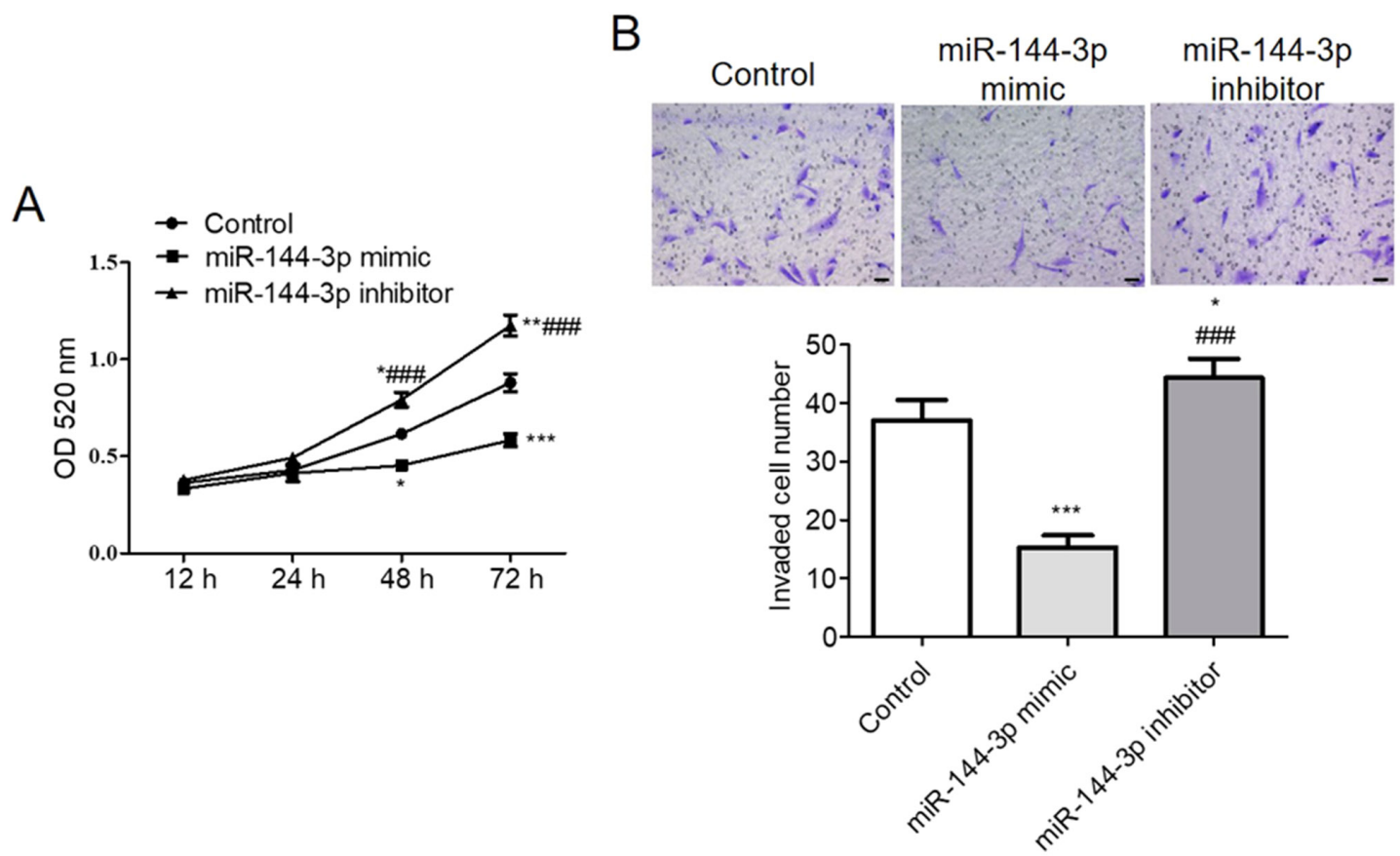

Figure 4. miR-144-3p overexpression decreases the malignancy of glioma cells. Following transfection with the miR-144-3p mimic or inhibitor, (A) Cell Counting Kit-8 assays and (B) Transwell assays were performed to measure cell viability and invasion of U251 cells, respectively. All data are presented as the mean \pm SEM $(n=4) .{ }^{*} \mathrm{P}<0.05,{ }^{* *} \mathrm{P}<0.01$ and ${ }^{* * *} \mathrm{P}<0.001$ vs. Control. ${ }^{\# \# \#} \mathrm{P}<0.001$ vs. miR-144-3p mimic. Scale bar, $20 \mu \mathrm{m}$. Control, non-transfected U251 cells; OD, optical density; miR, microRNA.

were significantly higher in the miR-144-3p + BCL6 group compared with those in the miR-144-3p group (Fig. 5A). In addition, cell viability and invasion were significantly increased in the miR-144-3p + BCL6 group, compared with those in the miR-144-3p group (Fig. 5B and C). Therefore, BCL6 overexpression could partly attenuate the inhibitory effects of miR-144-3p on the viability and invasion of U251 cells, suggesting that BCL6 serve an important role in miR-144-3p-mediated regulation of glioma cell proliferation and invasion.

Overexpression of miR-144-3p or BCL6 knockdown suppress tumor growth in vivo. The in vitro results suggested that miR-144-3p suppressed glioma cell proliferation and invasion by targeting BCL6. Therefore, the effects of the miR-144-3p/BCL-6 axis on glioma tumorigenesis was assessed further in vivo using xengraft nude mouse model transplanted with a subcutaneous glioma tumors. Following transfection with miR-144-3p mimic, miR-NC, BCL6-shRNA or NC-shRNA, U251 cells $\left(2 \times 10^{6}\right)$ were subcutaneous injected into the flanks of the mice. The tumor volumes were measured every week. The tumor size is shown in Fig. 6A. A significantly decreased average volume of tumors was observed in mice injected with cells transfected with either miR-144-3p mimic or cells transfected with BCL6-shRNA compared with those injected with either miR-NC or NC-shRNA, respectively (Fig. 6B). These results 


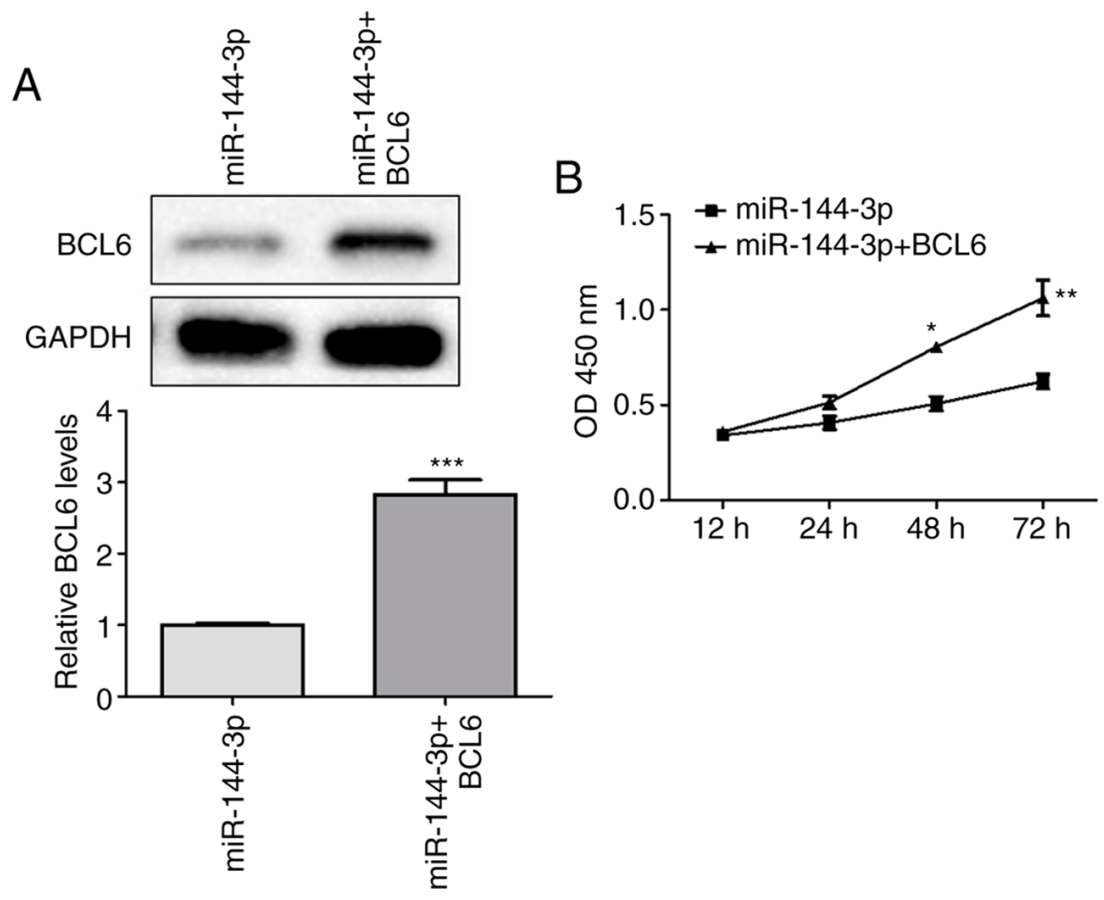

C
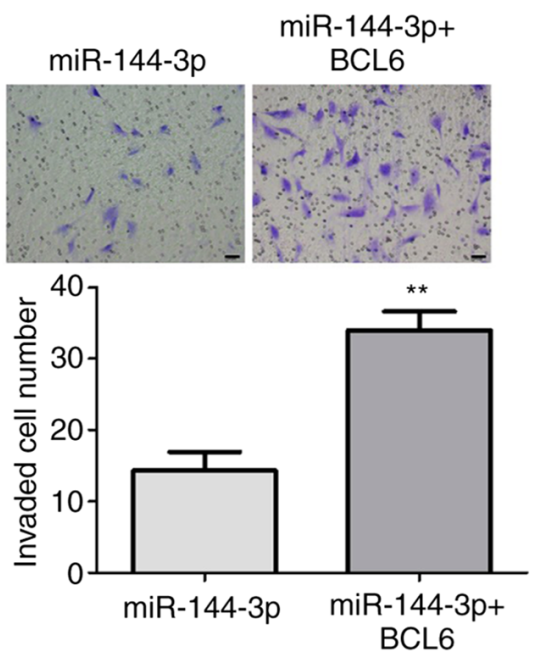

Figure 5. BCL6 plasmid co-transfection partly abolishes the inhibitory effects of miR-144-3p on U251 cell viability and invasion. U251 cells were co-transfected with the miR-144-3p mimic and BCL6-activation plasmid before (A) western blotting, (B) Cell Counting Kit-8 and (C) Transwell assays were performed to measure BCL6 expression, cell viability and cell invasion, respectively. ${ }^{*} \mathrm{P}<0.05,{ }^{* *} \mathrm{P}<0.01$ and ${ }^{* * * *} \mathrm{P}<0.001$ vs. miR-144-3p. Scale bar, $20 \mu \mathrm{m}$. miR, microRNA; OD, optical density.

A

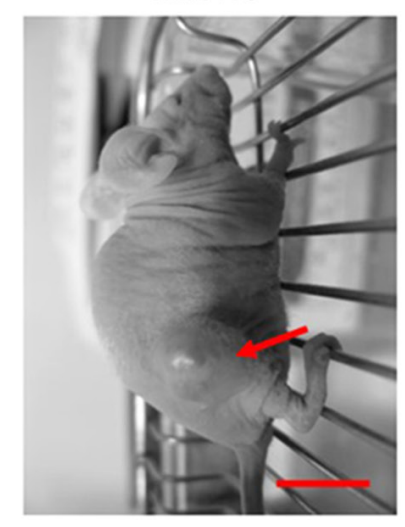

C

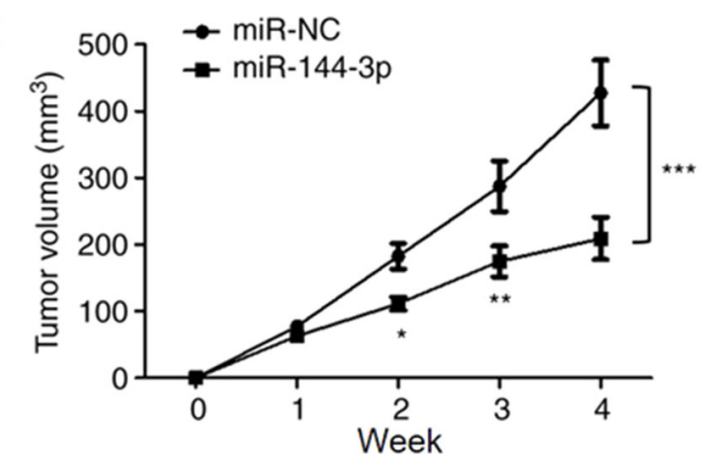

B
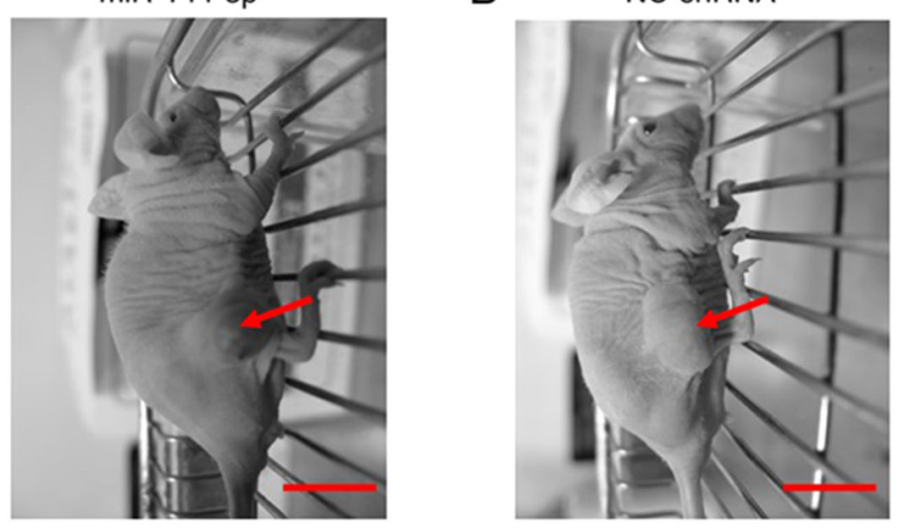

D

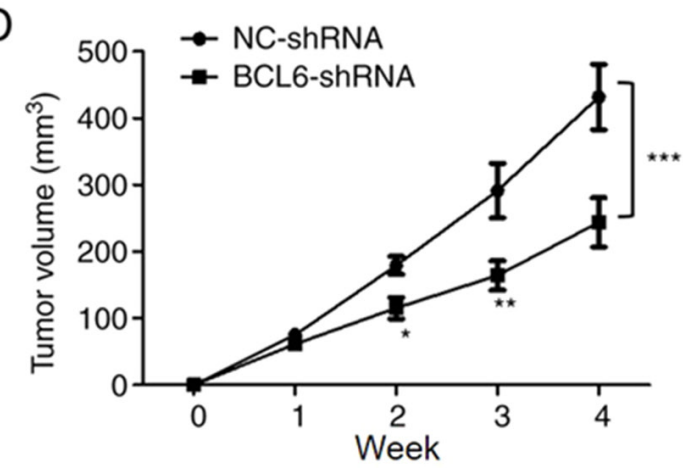

Figure 6. miR-144-3p upregulation or BCL knockdown suppress glioma tumor growth in vivo. Stably-transfected U251 cells were subcutaneously injected into the flanks of nude mice. In total, four groups of nude mice are shown with the corresponding tumor sizes 4 weeks following inoculation. Representative images of tumors (A) after injection of cells transfected with miR-NC or miR-144-5p mimic and (B) after injection of cell transfected with NC-shRNA or BCL6-shRNA. Tumor volumes (C) after injection of cells transfected with miR-NC or miR-144-5p mimic and (D) after injection of cell transfected with NC-shRNA or BCL6-shRNA were measured every week. Red arrows to indicate the location of the tumors on the mice. Scale bar, 10 mm. All data are presented as the mean $\pm \mathrm{SEM}, \mathrm{n}=6$ mice per group. ${ }^{*} \mathrm{P}<0.05,{ }^{* *} \mathrm{P}<0.01$ and ${ }^{* * *} \mathrm{P}<0.001$ vs. the NC (miR-NC or NC-shRNA) group. miR, microRNA; sh, short hairpin; NC, negative control. 
suggested that the miR-144-3p/BCL6 axis is involved in malignant glioma progression in vivo.

\section{Discussion}

BCL6 has been reported to to be a possible prognostic biomarker and potential therapeutic target that exhibits abnormal expression levels and serves a regulatory role in breast cancer, epithelial ovarian cancer and glioma (10-12). Specifically, BCL6 can serve as a potential indicator of tumor malignancy in breast cancer and glioma (10-12). It has been demonstrated that miRNA is an epigenetic regulator that targets the 3'-UTR sequence of target mRNAs and serves an important role in the development of several diseases (13). Increased miR-10b expression inhibits osteoblast differentiation by directly targeting the 3'-UTR sequence of BCL6 to increase the expression levels of STAT1 and block the nuclear translocation of Runx2, all of which were reversed by miR-10b downregulation (24). By contrast, inhibition of miR-10b induces osteoblast differentiation in a manner that was partially reversed by downregulating BCL6 expression (24). Furthermore, BCL6 has been shown to regulate miRNA levels and promotes lymphomagenesis by repressing miRNA-155 (25). In the present study, high levels of BCL6 were noted in glioma tissues. These findings are in agreement with those reported in a previous study by $\mathrm{Xu}$ et al (12), suggesting that BCL6 may be a tumor promoter for glioma pathogenesis and a valuable prognostic indicator in patients with glioma patients.

A number of studies have indicated that miR-144-3p is involved in a diverse range of biological functions, including cell proliferation, angiogenesis of lung vessels, oxidative stress and osteoporosis (26-28). The contribution of miR-144-3p in tumorigenesis is complex, where its role as a tumor physiology remains controversial. Previous studies have shown that miR-144-3p causes a decreased expression of target genes in various tumor tissues. miRNA-144-3p has been shown to interact with ETS-1 in laryngeal squamous cell carcinoma (29) but downregulates mTOR levels in salivary adenoid carcinoma (30). In addition, miR-144-3p can target enhancer of zeste 2 polycomb repressive complex 2 subunit in lung adenocarcinoma (31) whilst downregulating centrosomal protein 55 in prostate cancer (32). miR-144-3p has also been shown to regulates serum/glucocorticoid regulated kinase family member 3 function in hepatocellular carcinoma (33) but targets FOSB and proline rich protein 11 in pancreatic cancer (34). Furthermore, miR-144-3p can regulate c-MET in multiple myeloma (32) and inhibit tumorigenesis of oral squamous cell carcinoma by targeting the endoplasmic reticulum oxidoreductase $1 \alpha /$ STAT3 signaling pathway (35). miR-144-3p inhibits human neuroblastoma cell proliferation by regulating homeobox A7 expression (36). Jiang et al (37) previously demonstrated that miR-144-3p suppressed TGF- $\beta 1$-induced A549 cell malignancy by surpressing Src expression (37). Wu et al (38) reported that miR-144-3p expression was downregulated in cervical cancer tissues and cell lines, where miR-144-3p overexpression suppressed cancer cell malignancy. However, previous studies have also shown that miR-144-3p is involved in tumorigenesis. A previous study by Liu et al (39) found that miR-144-3p expression was increased in patients with papillary thyroid carcinoma, such that its overexpression promoted cell survival and cell cycle progression by targeting paired box gene 8 (39). In addition, miR-144-3p has been identified to be a novel plasma diagnostic indicator for clear cell renal cell carcinoma (ccRCC) (40). AT-Rich Interaction Domain 1A expression is suppressed by miR-144-3p upregulation, which induces ccRCC malignancy and mediates evasion of Sunitinib treatment (41). It has also been reported that miR-144-3p levels are upregulated in both peripheral blood and bone marrow samples of patients with acute myeloid leukemia compared with those in healthy individuals, whereas miR-144-3p targets nuclear factor, erythroid 2 like 2 in HL-60 cells (42). Song et al (43) reported that miR-144-3p facilitated the progression of nasopharyngeal carcinoma by mediating PTEN via crosstalk with the PI3K-AKT signaling pathway (43).

In addition, previous studies have demonstrated that miR-144-3p levels were significantly downregulated in glioma tissues compared with those in normal brain tissues, which were also decreased with tumor grade (44-46). miR-144-3p levels exhibited a positive correlation with overall survival of glioma patients (44). Following human cytomegalovirus (HCMV) infection, miR-144-3p levels are decreased whereas the expression of DNA Topoisomerase II $\alpha$ (TOP2A) increases (44). Increased miR-144-3p levels also inhibit clone formation, cell proliferation and invasion in HCMV-infected glioma cells by targeting TOP2A in vitro (44). In glioblastoma cells, the overexpression of miR-144-3p reduces the viability of cells and increases cell death, resulting in enhancement of the therapeutic effects of radiation and temozolomide (45). Frizzle class receptor 7 is another reported target of miR-144-3p-mediated suppression of glioma metastasis (46). Song et al (44) showed that the expression of miR-144-3p in HCMV-positive samples was markedly lower compared with that in HCMV-negative samples, where miR-144-3p expression correlated negatively with TOP2A expression. In addition, Lan et al (45) previously demonstrated that miR-144-3p expression is decreased in glioma tissues and associated with the OS of glioma patients. In this previous study (45), miR-144-3p exhibited a relatively high expression levels in non-neoplastic brain tissues whereas its expression was distinctly decreased in glioma tissues. In addition, there was also a significant difference in miR-44-3p expression between high grade (III-IV) and low-grade (I-II) glioma tissues. This decreased expression of miR-144-3p was associated with higher pathological grades, lower Karnofsky performance scores (45) and poorer survival rates (45). However, the latent role of miR-144-3p in glioma remains unclear. The present study demonstrated lower levels of miR-144-3p expression in 25 glioma patients, whilst overexpresseion of miR-144-3p notably suppressed cell proliferation and invasion of U251 cells. Upregulating BCL6 levels could reverse the inhibition caused by $\mathrm{miR}-144-3 \mathrm{p}$ overexpression. This occurred due to the direct targeting of the 3'-UTR sequence of BCL6 by miR-144-3p. The results indicated that BCL6 acted as a downstream interacting factor of miR-144-3p in glioma. $\mathrm{Xu}$ et al (12) revealed that BCL6 knockdown suppressed the tumorigenic phenotype of glioblastoma cells and induced cell degeneration (12). Therefore, miR-144-3p-mediated BCL6 inhibition may serve to be a potential strategy for treating glioma.

However, it should be noted that the lack of co-transfected cell xenograft experiments is a potential limitation of the 
present study. In addition, larger clinical samples sizes will need to be collected and conducted in future studies.

To conclude, the present study revealed that miR-144-3p and BCL6 were clinical predictors of patients with glioma and that the therapeutic potential of miR-144-3p/BCL6 axis should be exploited further for glioma treatment. Therefore, additional analysis of a higher number of samples and multiple grade tumors is required in the future to verify the diagnostic and prognostic potential of the miR-144-3p/BCL6 axis in glioma.

\section{Acknowledgements}

Not applicable.

\section{Funding}

No funding was received.

\section{Availability of data and materials}

The data used and/or analyzed in the current work are available from the corresponding author on reasonable request.

\section{Authors' Contributions}

REL and JRZ conceived and designed the study. JRZ carried out the experiments. REL and JRZ performed data analysis, wrote and revised the manuscript. All authors read and approved the manuscript.

\section{Ethics approval and consent to participate}

Research involving human participants and animal experiments was authorized by the Ethics Committee of Peking University People's Hospital (Beijing, China). The NIH Guide for the Care and Use of Laboratory Animals (the 8th edition) was followed. All procedures performed in the studies involving human participants were in accordance with the ethical standards of the Medical Ethics Expert Committee of National Health and Family Planning and adhered to the 1964 Helsinki declaration and its later amendments or comparable ethical standards. The study protocol was approved by the Ethical Committee of Peking University People's Hospital (Beijing, China). Written informed consent was obtained from all individual participants included in this work.

\section{Patient consent for publication}

Not applicable.

\section{Competing interests}

The authors declare that they have no competing interests.

\section{References}

1. Zhang Q, Xiang W, Yi DY, Xue BZ, Wen WW, Abdelmaksoud A, Xiong NX, Jiang XB, Zhao HY and Fu P: Current status and potential challenges of mesenchymal stem cell-based therapy for malignant gliomas. Stem Cell Res Ther 9: 228, 2018.

2. Alphandery E: Glioblastoma treatments: An account of recent industrial developments. Front Pharmacol 9: 879, 2018.
3. Towner RA and Smith N: In vivo and in situ detection of macromolecular free radicals using immuno-spin trapping and molecular magnetic resonance imaging. Antioxid Redox Signal 28: 1404-1415, 2018

4. Feng E, Liang T, Wang X, Du J, Tang K, Wang X, Wang F and You G: Correlation of alteration of HLA-F expression and clinical characterization in 593 brain glioma samples. J Neuroinflammation 16: 33, 2019.

5. Tompa M, Kalovits F, Nagy A and Kalman B: Contribution of the Wnt pathway to defining biology of glioblastoma. Neuromolecular Med 20: 437-451, 2018.

6. Yamini B: NF- $\kappa$ B, mesenchymal differentiation and glioblastoma. Cells 7: 125, 2018.

7. Brennan CW, Verhaak RG, McKenna A, Campos B Noushmehr H, Salama SR, Zheng S, Chakravarty D, Sanborn JZ, Berman SH, et al: The somatic genomic landscape of glioblastoma. Cell 155: 462-477, 2013.

8. Cancer Genome Atlas Research Network: Comprehensive genomic characterization defines human glioblastoma genes and core pathways. Nature 455: 1061-1068, 2008.

9. Sato M, Kanemoto H, Kagawa Y, Kobayashi T, Goto-Koshino Y, Mochizuki H, Takahashi M, Fujino Y, Ohno K and Tsujimoto H: Evaluation of the prognostic significance of BCL6 gene expression in canine high-grade B-cell lymphoma. Vet J 191: 108-114, 2012.

10. Ang L, Zheng L, Wang J, Huang J, Hu HG, Zou Q, Zhao Y, Liu QM, Zhao M and Wu ZS: Expression of and correlation between BCL6 and ZEB family members in patients with breast cancer. Exp Ther Med 14: 3985-3992, 2017.

11. Zhu L, Feng H, Jin S, Tan M, Gao S, Zhuang H, Hu Z, Wang H, Song Z and Lin B: High expressions of BCL6 and Lewis y antigen are correlated with high tumor burden and poor prognosis in epithelial ovarian cancer. Tumour Biol 39: 1010428317711655 , 2017.

12. Xu L, Chen Y, Dutra-Clarke M, Mayakonda A, Hazawa M, Savinoff SE, Doan N, Said JW, Yong WH, Watkins A, et al: BCL6 promotes glioma and serves as a therapeutic target. Proc Natl Acad Sci USA 114: 3981-3986, 2017.

13. Wei Z, Gao W, Wu Y, Ni B and Tian Y: Mutual interaction between BCL6 and miRNAs contributing to the pathogenesis of various cancers. Clin Transl Oncol 17: 841-846, 2015.

14. Zhu Z, Wang S, Zhu J, Yang Q, Dong $\mathrm{H}$ and Huang J: MicroRNA-544 down-regulates both Bcl6 and Stat3 to inhibit tumor growth of human triple negative breast cancer. Biol Chem 397: 1087-1095, 2016.

15. Sun C, Li S, Yang C, Xi Y, Wang L, Zhang F and Li D: MicroRNA-187-3p mitigates non-small cell lung cancer (NSCLC) development through down-regulation of BCL6. Biochem Biophys Res Commun 471: 82-88, 2016.

16. Li YY, Shao JP, Zhang SP, Xing GQ and Liu HJ: miR-519d-3p inhibits cell proliferation and invasion of gastric cancer by downregulating B-cell lymphoma 6 . Cytogenet Genome Res 154 : $12-19,2018$

17. Dragomir M, Mafra ACP, Dias SMG, Vasilescu C and Calin GA: Using microRNA networks to understand cancer. Int J Mol Sci 19: 1871, 2018.

18. Sun Z, Shi K, Yang S, Liu J, Zhou Q, Wang G, Song J, Li Z, Zhang Z and Yuan W: Effect of exosomal miRNA on cancer biology and clinical applications. Mol Cancer 17: 147, 2018.

19. Zhou Q, Liu J, Quan J, Liu W, Tan H and Li W: MicroRNAs as potential biomarkers for the diagnosis of glioma: A systematic review and meta-analysis. Cancer Sci 109: 2651-2659, 2018.

20. Wang S, Yin Y and Liu S: Roles of microRNAs during glioma tumorigenesis and progression. Histol Histopathol 34: 213-222, 2019.

21. Huang SW, Ali ND, Zhong L and Shi J: MicroRNAs as biomarkers for human glioblastoma: Progress and potential. Acta Pharmacol Sin 39: 1405-1413, 2018.

22. Koshkin FA, Chistyakov DA, Nikitin AG, Konovalov AN, Potapov AA, Usachyov DY, Pitskhelauri DI, Kobyakov GL, Shishkina LV and Chekhonin VP: Profile of microRNA expression in brain tumors of different malignancy. Bull Exp Biol Med 157: 794-797, 2014.

23. Livak KJ and Schmittgen TD: Analysis of relative gene expression data using real-time quantitative PCR and the 2(-Delta Delta C(T)) method. Methods 25: 402-408, 2001.

24. Yang J, Wang S, Wang F, Mu X, Qu Y, Zhao Z and Yu X: Downregulation of miR-10b promotes osteoblast differentiation through targeting Bc16. Int J Mol Med 39: 1605-1612, 2017. 
25. Basso K, Schneider C, Shen Q, Holmes AB, Setty M, Leslie C and Dalla-Favera R: BCL6 positively regulates AID and germinal center gene expression via repression of miR-155. J Exp Med 209: 2455-2465, 2012

26. Yang C, Lv K, Chen B, Yang Y, Ai X, Yu H, Yang Y, Yi B and Lu K: miR144-3p inhibits PMVECs excessive proliferation in angiogenesis of hepatopulmonary syndrome via Tie2. Exp Cell Res 365: 24-32, 2018.

27. Wang $\mathrm{C}, \mathrm{He} \mathrm{H}$, Wang L, Jiang $\mathrm{Y}$ and $\mathrm{Xu}$ Y: Reduced miR-144-3p expression in serum and bone mediates osteoporosis pathogenesis by targeting RANK. Biochem Cell Biol 96: 627-635, 2018.

28. Li Y, Zhao Y, Cheng M, Qiao Y, Wang Y, Xiong W and Yue W: Suppression of microRNA-144-3p attenuates oxygen-glucose deprivation/reoxygenation-induced neuronal injury by promoting Brg1/Nrf2/ARE signaling. J Biochem Mol Toxicol 32: e22044, 2018.

29. Zhang SY, Lu ZM, Lin YF, Chen LS, Luo XN, Song XH, Chen SH and Wu YL: miR-144-3p, a tumor suppressive microRNA targeting ETS-1 in laryngeal squamous cell carcinoma. Oncotarget 7: 11637-11650, 2016.

30. Huo F, Zhang C, He H and Wang Y: MicroRNA-144-3p inhibits proliferation and induces apoptosis of human salivary adenoid carcinoma cells via targeting of mTOR. Biotechnol Lett 38 409-416, 2016

31. Liu C, Yang Z, Deng Z, Zhou Y, Gong Q, Zhao R and Chen T: Downregulated miR-144-3p contributes to progression of lung adenocarcinoma through elevating the expression of EZH2. Cancer Med 7: 5554-5566, 2018

32. Zheng $H$, Guo Z, Zheng $X$, Cheng $W$ and Huang $X$ : MicroRNA-144-3p inhibits cell proliferation and induces cell apoptosis in prostate cancer by targeting CEP55. Am J Trans Res 10: 2457-2468, 2018.

33. Wu M, Huang C, Huang X, Liang R, Feng $Y$ and Luo $X$ : MicroRNA-144-3p suppresses tumor growth and angiogenesis by targeting SGK3 in hepatocellular carcinoma. Oncol Rep 38: 2173-2181, 2017.

34. Liu S, Luan J and Ding Y: miR-144-3p Targets FosB Proto-oncogene, AP-1 transcription factor subunit (FOSB) to suppress proliferation, migration, and invasion of PANC-1 pancreatic cancer cells. Oncol Res 26: 683-690, 2018.
35. Li X, Li Y, Jiang C, Chen L and Gan N: MicroRNA-144-3p inhibits tumorigenesis of oral squamous cell carcinoma by downregulating ERO1L. J Cancer 11: 759-768, 2020.

36. CaoXY,SunZY,ZhangLJ,Chen MKand YuanB:MicroRNA-144-3p suppresses human neuroblastoma cell proliferation by targeting HOXA7. Eur Rev Med Pharmacol Sci 23: 716-723, 2019.

37. Jiang $\mathrm{W}, \mathrm{Xu} \mathrm{Z}, \mathrm{Yu} \mathrm{L}$, Che J, Zhang J and Yang J: MicroRNA-144-3p suppressed TGF- $\beta 1$-induced lung cancer cell invasion and adhesion by regulating the Src-Akt-Erk pathway. Cell Biol Int 2019 (Epub ahead of print).

38. Wu J, Zhao Y, Li F and Qiao B: miR-144-3p: A novel tumor suppressor targeting MAPK6 in cervical cancer. J Physiol Biochem 75: 143-152, 2019.

39. Liu C, Su C, Chen Y and Li G: miR-144-3p promotes the tumor growth and metastasis of papillary thyroid carcinoma by targeting paired box gene 8. Cancer Cell Int 18: 54, 2018.

40. Lou N, Ruan AM, Qiu B, Bao L, Xu YC, Zhao Y, Sun RL, Zhang ST, Xu GH, Ruan HL, et al: miR-144-3p as a novel plasma diagnostic biomarker for clear cell renal cell carcinoma. Urol Oncol 35: 36.e7-36.e14, 2017.

41. Xiao W, Lou N, Ruan H, Bao L, Xiong Z, Yuan C, Tong J, Xu G, Zhou Y, Qu Y, et al: Mir-144-3p promotes cell proliferation, metastasis, sunitinib resistance in clear cell renal cell carcinoma by downregulating ARID1A. Cell Physiol Biochem 43: 2420-2433, 2017.

42. Sun X, Liu D, Xue Y and Hu X: Enforced miR-144-3p expression as a non-invasive biomarker for the acute myeloid leukemia patients mainly by targeting NRF2. Clin Lab 63: 679-687, 2017.

43. Song L, Chen L, Luan Q and Kong Q: miR-144-3p facilitates nasopharyngeal carcinoma via crosstalk with PTEN. J Cell Physiol 234: 17912-17924, 2019.

44. Song J, Ma Q, Hu M, Qian D, Wang B and He N: The Inhibition of miR-144-3p on cell proliferation and metastasis by targeting TOP2A in HCMV-positive glioblastoma cells. Molecules 23: 3259,2018

45. Lan F, Yu H, Hu M, Xia T and Yue X: miR-144-3p exerts anti-tumor effects in glioblastoma by targeting c-Met. J Neurochem 135: 274-286, 2015.

46. Cheng ZX, Song YX, Wang ZY, Wang Y and Dong Y: miR-144-3p serves as a tumor suppressor by targeting FZD7 and predicts the prognosis of human glioblastoma. Eur Rev Med Pharmacol Sci 21: 4079-4086, 2017. 